\title{
INGREDIENTES PEDAGÓGICOS PARA PREPARAR FUTUROS DOCENTES DE COCINA
}

\author{
Doris Guadalupe Cornejo Cabrera \\ Universidad Internacional Iberoamericana (México) \\ lupibeiro@yahoo.com.br \\ Ma Dolores Molina Jaén \\ Centro Universitario SAFA Úbeda (España) \\ mdmolina@fundacionsafa.es · https://orcid.org/0000-0001-6010-6382
}

\begin{abstract}
Resumen. Uno de los temas más controvertidos relacionados con la Educación Superior es la formación del futuro docente, tópico que enmarca el presente estudio. Nuestro propósito ha sido investigar elementos del entorno estudiantil y docente para poder describir la realidad en cuanto a la aplicación de técnicas didácticas por parte del docente e identificar las condiciones que rodean el desarrollo de aprendizajes relacionados en el grado de Técnico Superior en Gastronomía, en la Escuela de Gastronomía en El Salvador, como efecto del acto didáctico del docente que tiene formación Superior en Educación y del docente que no la tiene. Se han diseñado dos instrumentos o Escalas Likert, que siendo validadas por estudiantes universitarios y profesionales expertos en Educación y Turismo Gastronómico, fueron estructuradas en apartados correspondientes con las dimensiones de la investigación. Todos los sujetos de las muestras de estudiantes han sido elegidos aleatoriamente y la muestra de docentes se corresponde con el universo poblacional de los docentes que imparten asignaturas en la Escuela de Gastronomía. Una vez aplicada una metodología descriptiva y bajo los análisis estadísticos correspondientes, se evidencia la necesidad de aplicar técnicas didácticas específicas partiendo de la experiencia previa del estudiante y la necesidad de implementar un programa permanente de formación docente que oriente su intervención didáctica para crear experiencias en las aulas a fin de favorecer el aprendizaje en este alumnado.
\end{abstract}

Palabras clave: Didáctica, docente, gastronomía.

\section{PEDAGOGICAL INGREDIENTS TO PREPRARE FUTURE KITCHEN TEACHERS}

\begin{abstract}
One of the most controversial issues related to Higher Education is the training of the future teacher, a topic that frames the present study. Our purpose has been to investigate elements of the student and teaching environment in order to describe the reality regarding the application of teaching techniques by the teacher and identify the conditions that surround the development of related learning in the degree of Superior Technician in Gastronomy, in the School of Gastronomy in El Salvador, as an effect of the didactic act of the teacher who has a Superior Education formation and of the teacher who does not have it. Two instruments or Likert Scales have been designed, which, being validated by university students and professionals specialized in Education and Gastronomic Tourism, were structured in sections corresponding to the dimensions of the research. All the subjects of the student samples have been chosen randomly and the sample of teachers corresponds to the population universe of the teachers who teach subjects in the School of Gastronomy. Once a descriptive methodology has been applied and under the corresponding statistical analysis, it is evident the need to apply specific didactic techniques starting from the student's previous experience and the need to implement a permanent teacher training program that guides their didactic intervention to create experiences in the classrooms in order to promote learning in this student body.
\end{abstract}

Keywords: Teacher, gastronomy, didactics. 


\title{
INGREDIENTES PEDAGÓGICOS PARA PREPARAR FUTUROS PROFESSORES DE COZINHA
}

\begin{abstract}
Resumo. Uma das questões mais controversas relacionadas à Educação Superior é a formação do futuro professor, um tópico que enquadra o presente estudo. Nosso objetivo tem sido investigar elementos do estudante e do ambiente de ensino, a fim de descrever a realidade sobre a aplicação de técnicas de ensino pelo professor e identificar as condições que cercam o desenvolvimento da aprendizagem relacionada no grau de Superior Técnico em Gastronomia, no Escola de Gastronomia em El Salvador, como efeito do ato didático do professor que tem formação em Educação Superior e do professor que não tem. Foram elaborados dois instrumentos ou escalas Likert, que, validados por universitários e profissionais especializados em Educação e Turismo Gastronômico, foram estruturados em seções correspondentes às dimensões da pesquisa. Todos os sujeitos das amostras dos alunos foram escolhidos aleatoriamente e a amostra de professores corresponde ao universo populacional dos professores que lecionam disciplinas na Escola de Gastronomia. Uma vez aplicada uma metodologia descritiva e sob análise estatística correspondente, fica evidente a necessidade de aplicar técnicas didáticas específicas a partir da experiência prévia do aluno e a necessidade de implementar um programa permanente de capacitação docente que orienta sua intervenção didática na criação de experiências na área. salas de aula, a fim de promover a aprendizagem neste corpo discente.
\end{abstract}

Palavras-chave: Professor, gastronomia, didática.

\section{Introducción}

Nuestra investigación tiene como objetivo principal analizar la formación superior del profesorado del nivel de Educación Superior que va a ser evaluado por un tipo de alumnado que ya tiene mucha experiencia académica, es joven adulto y es quién recibe la acción de dicho profesorado.

Esa formación del nivel Superior que debería tener el profesorado va encaminada en primer lugar, hacia su desarrollo profesional en particular, por lo que Yánez (2017, p.6) enfatiza que "sólo con el talento humano y el conocimiento se podrá crear riqueza". Su propuesta es un modelo educativo con la visión de formar personas no sólo basado en la transferencia de contenido sino más bien, debe sentar las bases en desarrollar al futuro profesional con el perfil necesario para que se pueda insertar de forma exitosa en el campo laboral y en este caso, con los estudiantes de profesorado que más tarde, pasarán a ser docentes.

El docente que imparte clases en las carreras de profesorado de nivel superior, necesita desarrollar competencias y desempeños profesionales para hacer frente a las nuevas necesidades de la Educación Superior y que van enfocadas en dos aspiraciones relevantes vinculadas que son, por una parte mejorar la formación pedagógica y didáctica del futuro profesor en sus proyectos curriculares de las carreras profesionales y por otra, las vinculadas con el conocimiento didáctico del contenido CDC (Porro, 2015).

Los centros universitarios y el profesor como figura pedagógica, continúan siendo autoridades en el campo de la traducción pedagógica del conocimiento para facilitar la comprensión del contenido y el desarrollo de habilidades en sus estudiantes. Esto se explica, en primer lugar, por la necesidad de un enfoque profesional y práctico para la formación del contenido de las clases, que debe ser siempre diverso y cumplir 
con las orientaciones prácticas para los alumnos; Para Mirzagitova \& Akhmetov (2016), los docentes son responsables de la precisión, integridad y adecuación del conocimiento que se transmite, así como de la relevancia de las competencias en formación. En segundo lugar, es importante comprender y aceptar la responsabilidad de la formación desde una cosmovisión histórica que permita a los alumnos evaluar críticamente la utilidad de lo trabajado. Podemos decir que un conocimiento sin causa de aplicación es lo mismo que una aplicación sin conocimiento de causa, es decir estaría incompleto.

El futuro docente construye su práctica por el conjunto de experiencias acumuladas a lo largo de toda su vida escolar como estudiante de los diversos niveles de formación. Siendo así, tiene un repositorio tan extenso como cargado de emotividad que incide, notablemente, en el desarrollo de modelos de acción cuando no existen otros referentes con mayor validez que puedan legitimar la actuación de los estudiantes en proceso de formación docente (Pavo y Ángel, 2014). Es así como el proceso de formación del futuro docente debería considerar esta experiencia académica previa.

\section{La didáctica y sus aportes a los problemas de metodología docente}

El proceso de la enseñanza siempre es una cuestión basada en dos elementos que son: con qué enseñar y a quién enseñar y Marhuenda y Ros-Garrido, 2015 identifican que la acción de los maestros depende de estas dos variables externas a ellos. Es así que Torres (2017), considera necesario romper la relación epistemológica de la enseñanza y el aprendizaje que se da en la Didáctica general y parte, desde la Didáctica Normativa, la cual orienta y guía al docente sobre cómo proceder dentro del aula y avanzar desde una visión formativa, hacia las Didácticas Especiales, las cuales van enfocadas a los campos específicos de las distintas ciencias del saber.

La Didáctica se basa en un replanteamiento crítico de la acción docente que puede llegar a superar las dificultades existentes en el aula, además de dar calidad al proceso de enseñanza aprendizaje (Sauthier y Dos Santos, 2016), pero vista bajo este enfoque, debe ofrecer las condiciones para la realización plena y verdadera de la docencia.

Si cada disciplina tiene una forma propia de proceder, con especiales y variadas técnicas que representan el saber hacer disciplinar; se destaca aquí la enorme necesidad de formar al docente desde las particularidades internas de la disciplina, en el sentido de que identifique las mejores formas de actuar para lograr los objetivos de aprendizaje que le exige la disciplina en particular (Bosch, 2000).

\section{La lógica de formar al docente en el uso de la Didáctica para el aula}

La lógica de la Didáctica es la lógica de la enseñanza. Sin embargo y de forma contradictoria, esta vocación lógica de la didáctica se realiza solamente por medio del aprendizaje de los sujetos participantes en el proceso, por lo tanto, el enfoque de la didáctica se hace más complejo porque se considera no solo el contenido de enseñanza, sino también el proceso y condiciones en las que se produce el aprendizaje y que, en su totalidad, representan el proceso de enseñanza.

Para Franco y Pimenta (2016), el foco de la didáctica en este proceso de enseñanza moviliza a los sujetos para elaborar la construcción y reconstrucción de los conocimientos y saberes. Según los autores elaborar la enseñanza implica organización de la transmisión de conocimientos siendo hoy más complejo si cabe porque el enfoque implica desencadenar en los estudiantes actividades intelectuales que les permitan crear sentido a los aprendizajes y así, reelaborarlos y transformarlos en saberes. 
Visto así, el docente de Educación Superior, como en cualquier otro nivel de enseñanza, necesita obviamente del dominio profundo de los conocimientos del área que enseña o conocimiento disciplinar, pero también se requieren competencias y habilidades didáctico-pedagógicas suficientes para hacer el aprendizaje más eficiente, tener una visión del mundo, del ser humano, de la ciencia y de la educación que son compatibles con la naturaleza de su cargo. En esta línea, Antoli (2016), ha identificado que la figura del futuro profesor encuentra una serie de dificultades, a veces debidas a los errores en su preparación y otras debido a la experiencia propiamente dicha y propone una fase de formación inicial que tenga en cuenta los aspectos que se muestran en Figura 1.
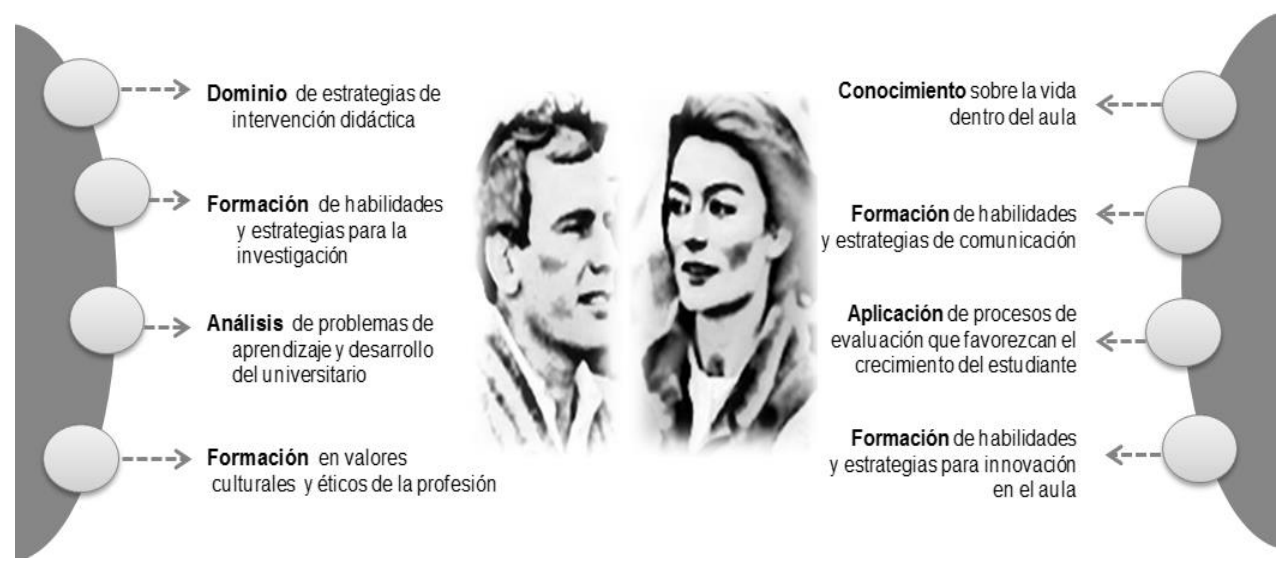

Figura 1. Fases de formación inicial del futuro profesor (Antoli , 2016)

Este planteamiento viene a representar un cambio sin precedentes en la docencia universitaria porque según De Oliveira y De Moura (2016), si el objetivo final de la educación es el aprendizaje, es a partir de ello que debe evaluarse a los alumnos, a los docentes y a las universidades. En ello radica la urgente necesidad de la formación didáctica del docente para el proceso educativo, en el cual el aprendizaje debe ser el fin último del quehacer de la docencia universitaria. El alumnado universitario, además, no funciona bajo modelos conductistas y tampoco es un adulto maduro como ha señalado Pacheco (2013, p. 29), es un alumnado conocido como "joven-adulto" ya que, estos jóvenes adultos están en una fase en la que deberían priorizar el aprender a aprender. Paradójicamente, si queremos ser docentes del nivel Superior, primero tenemos que aprender cómo aprenden los universitarios actuales.

\section{Investigar la Didáctica antes de aplicarla en el aula}

La Didáctica Específica como área de oportunidad para profesionalizar los programas de formación inicial de los docentes ha sido recientemente estudiada llegando a la conclusión de la urgente de especialización que requiere la formación del profesorado. A modo de ejemplo, algunos estudios que subrayan esta idea son:

- Didáctica y docencia en el área de Modas: Iszoro (2015), de la Universidad Politécnica de Madrid, un estudio que demuestra que en los Métodos directos de patronaje creativo se prioriza la experimentación.

- Didáctica aplicada al área de Música o Armonía, donde Devesa (2013), de la Universitat d'Alacant-Universidad de Alicante, estudia la Didáctica de la armonía como una propuesta de enseñanza basada en el aprendizaje significativo. 
- El reto docente aplicando Didáctica en la enseñanza del idioma español a estudiantes chinos (Zhou, 2015), estudio que revela cuales son las áreas de dificultad en el aprendizaje de las pasivas del español como lengua extranjera para estudiantes chinos y en qué aspectos se tiene que poner mayor énfasis a la hora de enseñarlo, proponiendo ejercicios específicos con los que los estudiantes tomen consciencia de los posibles errores y, por consiguiente, lleguen a evitarlos hasta lograr el aprendizaje.

- Didáctica en la interpretación del Arte en Museos (Benito, 2014), estudio exploratorio desarrollado desde la Universidad de Barcelona sobre la interpretación didáctica del arte en el museo a través de tecnologías móviles. La investigación presenta la museografía, museología, arte y educación vinculada con las características propias del Mobile learning como parte de la propuesta para el aprendizaje.

\section{Método}

\section{Diseño del trabajo}

Esta investigación ha utilizado el método de investigación científica, con fuerte sustento en la revisión de investigaciones precedentes que ayudaron a desarrollar el análisis de la situación a investigar y llegar a la comprensión de los hallazgos obtenidos. Se desarrolló con enfoque cuantitativo, lo cual permite analizar la realidad que se vive en la institución objeto de la investigación.

En base al trabajo de Gómez (2009, citado en Ballesteros 2016), se hizo la recolección y sistematización de datos con el objetivo de responder las preguntas de investigación planteadas: ¿es necesaria una didáctica universitaria específica en la promoción de competencias relacionadas con el Turismo Cultural Gastronómico en el campo de la formación Técnica Superior en Gastronomía?,¿se aplica una didáctica específica en la labor de los docentes con y sin formación pedagógica para impulsar aprendizajes relacionados con Turismo Cultural Gastronómico en alumnos de la carrera Técnica Superior en Gastronomía? y en este sentido, ¿cuál sería la didáctica adecuada para desarrollar aprendizajes relacionados a Turismo Gastronómico en la carrera Técnica Superior en Gastronomía?

Considerando los diversos ángulos bajo los cuales se puede hacer el estudio, la investigación se diseñó en 5 dimensiones o apartados y para el presente artículo únicamente se presenta la dimensión relacionada con las Estrategias metodológicas para el desarrollo de una formación adecuada en la Especialidad de Gastronomía estableciendo análisis con las variables mediadoras de formación pedagógica del docente.

Para la muestra de estudiantes, la dimensión fue estudiada en el apartado-C del instrumento diseñado, en el cual se plantearon 20 ítems o afirmaciones. Para la muestra de docentes se investigó en el apartado-D con el planteamiento de 14 ítems o afirmaciones, conforme se muestra la Tabla 1, en la cual se presenta el comparativo de los ítems o afirmaciones que se plantearon a cada una de las muestras. 
Tabla 1

Relación de ítems dimensión Estrategias metodológicas para el desarrollo de una formación adecuada en la especialidad de Gastronomía

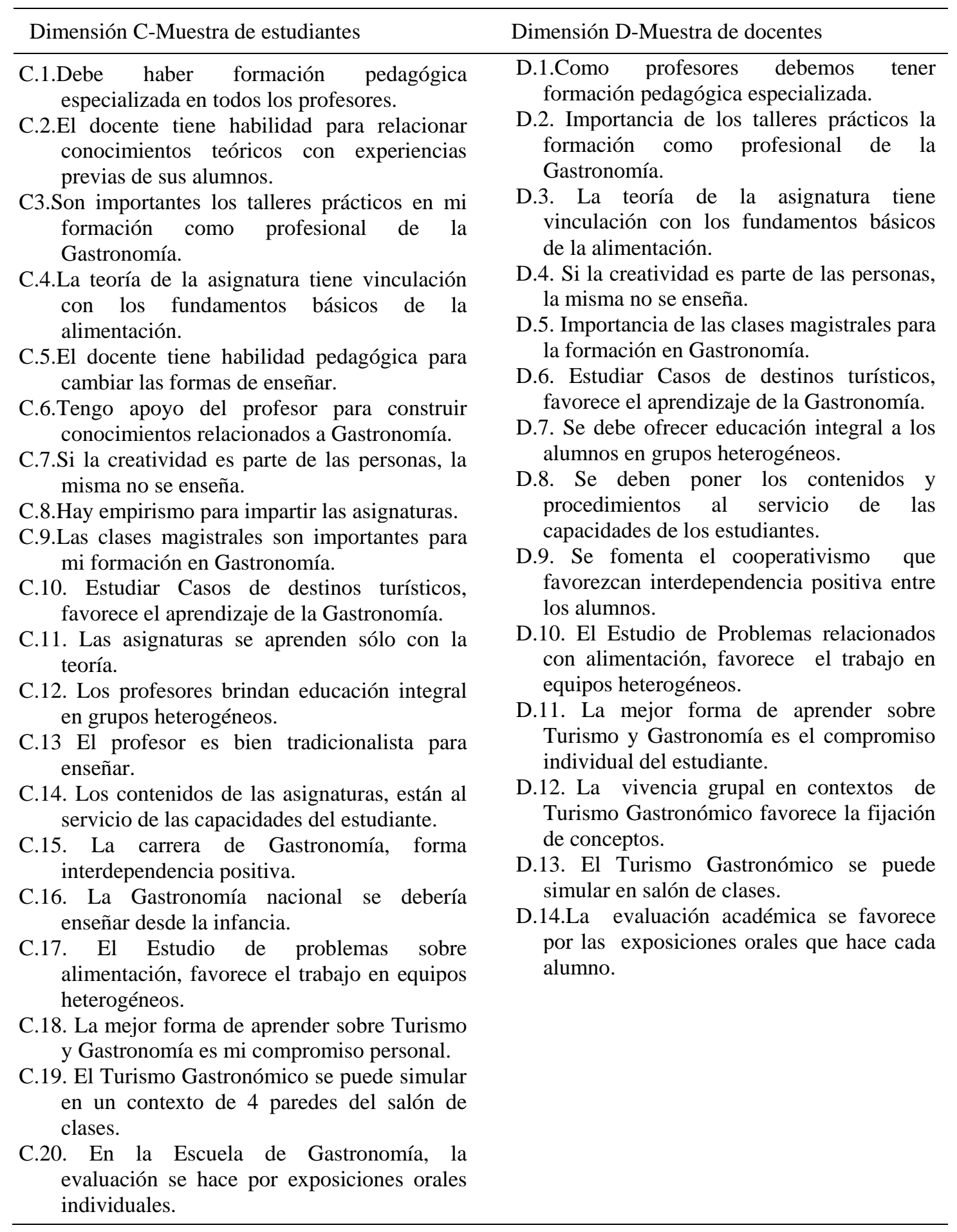

\section{Participantes y contexto}

Los participantes de la investigación son los actores claves del proceso de formación: estudiantes y docentes. Las muestras de sendos colectivos se conformaron con base en los siguientes criterios: 
- Los estudiantes fueron seleccionados de forma aleatoria. La muestra de docentes ha estado conformada por todos los docentes que imparten clases en la Escuela de Gastronomía dentro de la institución objeto de la presente investigación.

- Tanto los alumnos como los docentes debían estar activos en su rol académico dentro de la Escuela de Gastronomía y específicamente en el primer semestre del año 2018.

- $\quad$ Los sujetos de la muestra de docentes debían estar impartiendo asignaturas en la Escuela de Gastronomía de ITCA/ FEPADE.

- Los sujetos de la muestra de estudiantes, pertenecientes a un universo/población de 400 estudiantes, debían estar matriculados y cursando asignaturas en el periodo de la consulta y haber cursado, por lo menos, un ciclo o semestre de estudio.

- El tamaño de la muestra de estudiantes fue determinado considerando el nivel de confianza, error máximo, probabilidad de éxito y fracaso identificando que 195 sujetos debía ser el tamaño de la muestra. Tamaño determinado con base en la Asociación de Psicología Americana (APA, 2016) utilizando la ecuación que a continuación se muestra:

$$
n=\frac{N \times Z_{a}^{2} x p \times q}{d^{2} x(N-1)+Z_{a}^{2} x p \times q}
$$

Siendo:

$\mathrm{N}=$ tamaño de la población

$\mathrm{Z}=$ nivel de confianza

$\mathrm{p}=$ probabilidad de éxito o proporción esperada $\mathrm{q}=$ probabilidad de fracaso

$\mathrm{d}=$ precisión (Error máximo admisible en términos de proporción)

$$
n=\frac{400 \times 1.96^{2} \times 0.5 \times 0.5}{0.05 \times(400-1)+1.96^{2} \times 0.5 \times 0.5}
$$

De la ecuación se obtiene que la muestra de estudiantes debe ser de 195 sujetos; aunque la muestra final estuvo conformada por 202 estudiantes.

Con el grupo de docentes que forman la muestra y que son el universo, se analizaron dos grandes grupos: docentes que tienen formación de nivel Superior en Educación y docentes que no la tienen, lo cual se encuentra en la Figura 2, destacando, además, el tiempo de años de experiencia docente. 


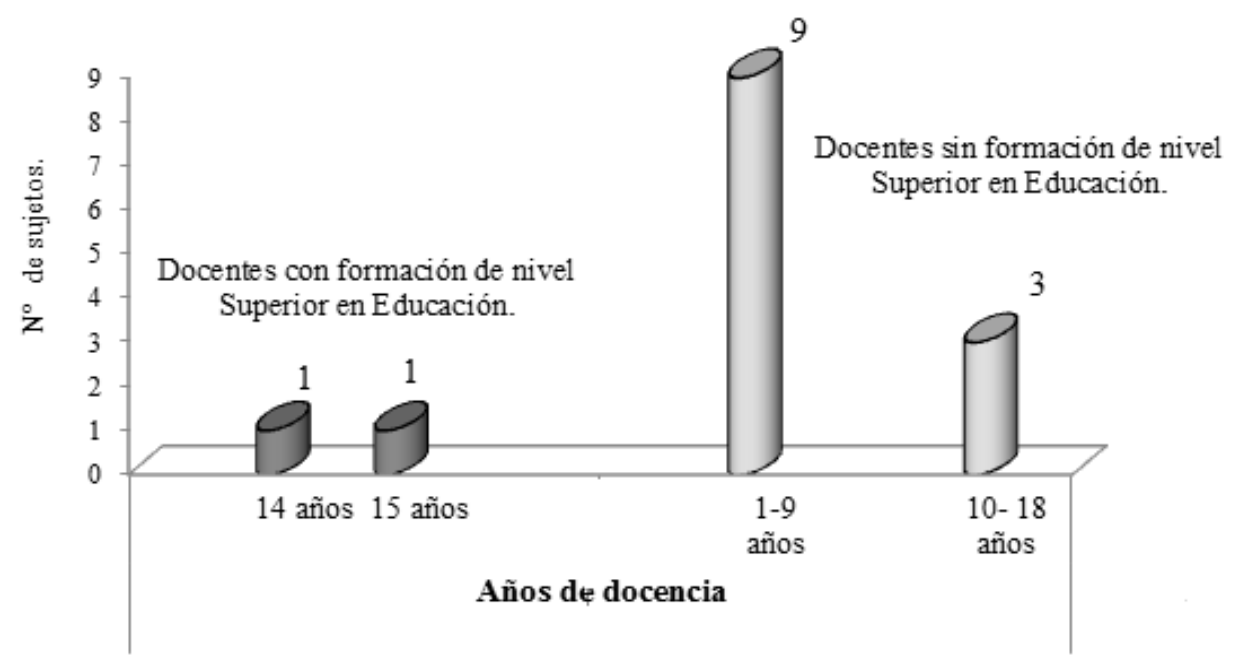

Figura 2. Distribución de profesores por cantidad de años de trabajo docente

A partir de este criterio de identificación del profesorado, se procedió a ordenar a sus estudiantes, siendo que de los 202 sujetos que componen la muestra, se identificaron que $\mathrm{n}=72$ alumnos tienen como docentes a profesorado con formación Superior en Educación y que $n=130$ alumnos tienen como docentes a profesorado sin formación superior en educación (Véase Figura 3).

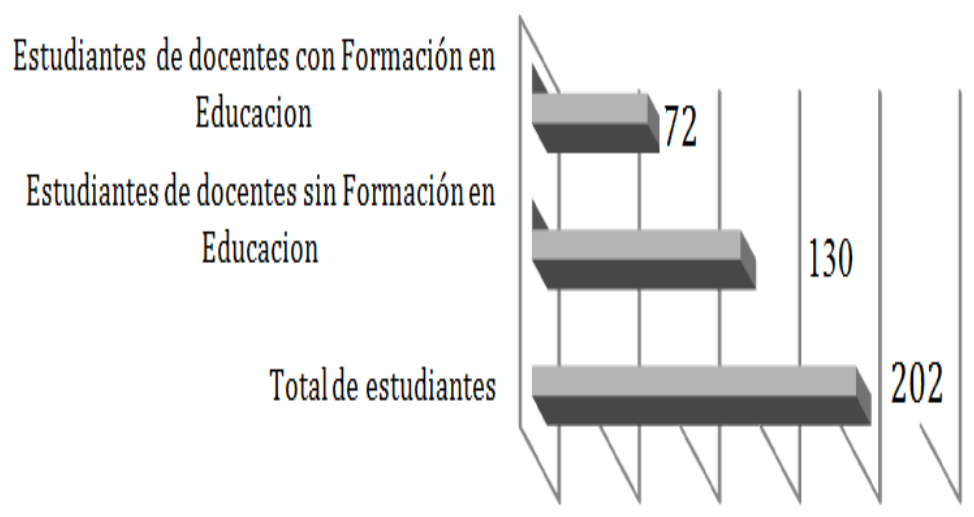

Figura 3. Distribución de los sujetos pertenecientes a la muestra de estudiantes

Es interesante destacar como características de la muestra de estudiantes que se trata de sujetos muy jóvenes con un rango de edades de entre 17 y 21 años, y que presenta poca diferencia en cuanto al género (Figura 4). 


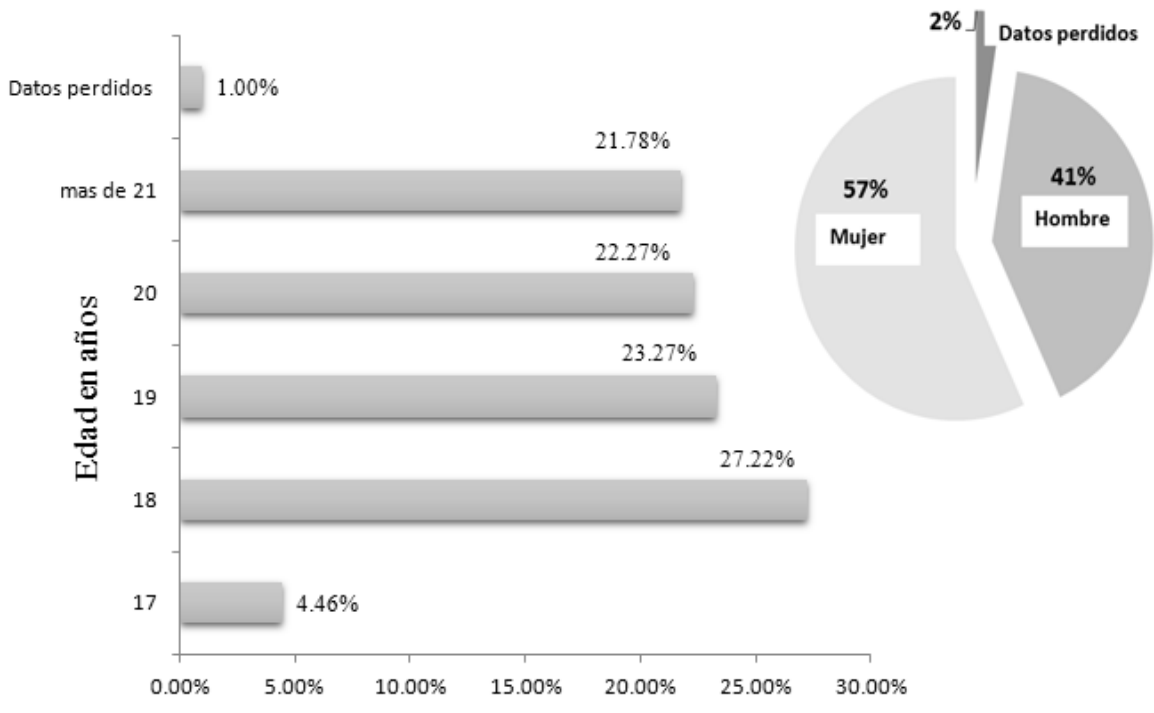

Figura 4. Distribución porcentual por edades y género de los sujetos pertenecientes a la muestra de estudiantes.

En cuanto al contexto de la investigación, la misma fue desarrollada en un solo centro de formación del sector público con experiencia de 50 años en la formación Técnica nivel de Educación Superior. En la selección de la muestra destacamos la importancia que reviste la perspectiva de los actores dentro de un fenómeno social como lo es el proceso de enseñanza-aprendizaje objeto de la presente investigación.

\section{Instrumentos de recogida de información}

La consulta la pudimos desarrollar haciendo uso de instrumentos diseñados para tal fin con estructura de escala de Likert expresando el grado de desacuerdo/acuerdo, oscilando las respuestas entre 1 y 4 (siendo 1 , en desacuerdo; 2 , poco; 3, bastante y 4 , totalmente de acuerdo). Los instrumentos planteados son dos: uno para alumnos, el cual tiene 77 ítems organizados en 4 dimensiones o apartados que responden a las dimensiones de estudio; y uno para docentes, con 61 ítems, organizado en 5 dimensiones o apartados. Los instrumentos fueron aplicados dentro de la institución de Educación Superior objeto de la presente investigación.

Para dar validez de contenido a los instrumentos, se utilizó la técnica de juicio de expertos. Para optimizar estos aportes se contó con la participación de dos profesionales salvadoreños con experiencia de 15 y 25 años en docencia y Dirección de Centros Escolares públicos respectivamente; así como también, de dos grupos de alumnos y sus docentes de Educación Superior en Turismo y dos profesionales del sector Turismo con experiencia nacional e internacional en la región centroamericana, a quienes se solicitó emitir su valoración del instrumento a efecto de mejorarlo.

Contrastamos la variabilidad entre los ítems y para conocer tanto la confiabilidad lograda por los dos instrumentos de medición, hicimos la prueba de coeficiente de alfa Cronbach. Únicamente se presentan las dimensiones C y D (estudiantes y profesorado) referidas a las estrategias metodológicas para el desarrollo de una formación adecuada en la especialidad de Gastronomía. Ambas presentan coeficientes con variaciones que identifican una elevada confiabilidad y no detecta redundancia de los ítems, siendo los valores de 0.811 y 0.786 para estudiantes y profesorado, respectivamente. 
Como se ha mencionado, todos los sujetos participantes son de la misma institución y la aplicación de los instrumentos se realizó presencialmente, con los estudiantes dentro de sus salones de clase y con los docentes la aplicación fue individual, cada uno en su oficina.

\section{Análisis de datos}

Las pruebas estadísticas se aplicaron a los datos recabados con sendos instrumentos, siendo que únicamente estamos presentando las pruebas relacionadas la dimensión C-Estrategias metodológicas para el desarrollo de una formación adecuada en la especialidad de gastronomía del instrumento para estudiantes. Del instrumento de los docentes se presentan dos dimensiones: la C-Relación de la Gastronomía y el Turismo Cultural y la D-Estrategias metodológicas para el desarrollo de una formación adecuada en la especialidad de Gastronómica (Tabla 2).

El procesamiento de datos se hizo usando la herramienta informática SPSS por ser paquete estadístico para la Ciencias Sociales, habiendo considerado tanto las medidas descriptivas de frecuencia, tendencia central y variabilidad, como también, las de inferencia de análisis paramétrico.

\section{Tabla 2}

Matriz correlación de componentes principales y medidas estadísticas significativas de dimensiones estrategias metodológicas para el desarrollo de una formación adecuada en la especialidad de Gastronomía y relación de Gastronomía y Turismo.

\begin{tabular}{|c|c|c|c|}
\hline \multirow{3}{*}{$\begin{array}{l}\text { Pruebas estadísticas aplicadas } \\
\text { en la investigación }\end{array}$} & \multicolumn{3}{|c|}{ Dimensión en los instrumentos } \\
\hline & \multirow{2}{*}{$\begin{array}{c}\begin{array}{c}\text { De } \\
\text { estudiantes }\end{array} \\
\mathrm{C}\end{array}$} & \multicolumn{2}{|c|}{$\begin{array}{c}\mathrm{De} \\
\text { docentes }\end{array}$} \\
\hline & & $\mathrm{C}$ & D \\
\hline $\begin{array}{l}\text { Descriptivas: } \\
\text { Distribución de frecuencias, medidas de tendencia central y de } \\
\text { variabilidad. Otras Medidas: Asimetría, Curtosis }\end{array}$ & $\checkmark$ & $\checkmark$ & $\checkmark$ \\
\hline $\begin{array}{l}\text { Fiabilidad y validez del instrumento: } \\
\text { Medida de consistencia interna: alfa de Cronbach }\end{array}$ & $\checkmark$ & $\checkmark$ & $\checkmark$ \\
\hline $\begin{array}{l}\text { Medidas de inferencia: } \\
\text { Análisis paramétricos: Coeficiente de correlación de Pearson, } \\
\text { Prueba t. } \\
\text { Análisis de varianza unidireccional (ANOVA one way). } \\
\text { Análisis no paramétricos: Coeficientes de correlación por rangos } \\
\text { ordenados de Spearman y Kendall. }\end{array}$ & $\checkmark$ & $\checkmark$ & $\checkmark$ \\
\hline $\begin{array}{l}\text { Prueba de Análisis factorial: } \\
\text { Método de rotación de componentes principales. Promax con } \\
\text { normalización Káiser }\end{array}$ & $\checkmark$ & $\checkmark$ & \\
\hline
\end{tabular}

\section{Resultados}

Inicialmente, presentamos los datos correspondientes a la muestra de estudiantes y específicamente a la dimensión que representa las condiciones que rodean la formación en la especialidad de Gastronomía de lo cual, consideramos destacable mencionar la experiencia previa que han tenido los estudiantes, en cuanto a cocinar con 
las personas mayores de su familia, como es la mamá, el papá o los abuelos, (Tabla 3), dado que muchas de las tradiciones culinarias son transferibles de forma oral.

Tabla 3

Experiencia previa que han tenido los estudiantes de la muestra cocinando con las personas mayores de su familia

\begin{tabular}{cccc}
\hline \multirow{2}{*}{ Sujetos de la muestra de estudiantes } & \multicolumn{2}{c}{ Experiencia previa del estudiante cocinando } \\
\cline { 2 - 4 } & Con la mamá & Con el papá & Con los abuelos \\
\hline Hombre & $38.12 \%$ & $16.83 \%$ & $26.24 \%$ \\
Mujer & $52.97 \%$ & $24.75 \%$ & $43.07 \%$ \\
\hline
\end{tabular}

A partir de los datos anteriores, encontramos a un alumnado que trae a la Escuela experiencias culinarias, por lo que hemos buscado conocer si a estos alumnos les gusta o no les gusta cocinar, en virtud que tanto la experiencia previa que se tiene y el disfrute por lo que se hace, son elementos que sirven de referencia para el ejercicio de la docencia en Escuelas de Gastronomía (Tabla 4).

Tabla 4

Distribución porcentual de género de los estudiantes expresando preferencia por el gusto a la cocina

\begin{tabular}{|c|c|c|c|c|}
\hline \multirow{2}{*}{ Criterio } & \multicolumn{2}{|c|}{ Género masculino } & \multicolumn{2}{|c|}{ Género femenino } \\
\hline & Fr & $\%$ acumulado & $\mathrm{Fr}$ & $\%$ acumulado \\
\hline \multicolumn{5}{|l|}{ Le gusta cocinar } \\
\hline SI & 81 & $98.78 \%$ & 112 & $97.39 \%$ \\
\hline NO & 1 & $1.22 \%$ & 3 & $2.61 \%$ \\
\hline No respondieron & 5 & $100.00 \%$ & 0 & $100.00 \%$ \\
\hline
\end{tabular}

Este escenario viene a plantear un gran reto en el ejercicio de la docencia, por lo que también se ha buscado conocer de los docentes esta identificación con la cocina y nos referimos al ítem que busca conocer si los docentes les gusta cocinar o no y encontramos que al $64.29 \%$ de los docentes expresan que, si le gusta, en cuanto que el $35.71 \%$ expresan que no les gusta cocinar.

En cuanto a la experiencia culinaria que han tenido estos docentes habiendo cocinado con la mamá, los datos relejan que es mayor para las mujeres docentes $(75 \%)$ que los docentes hombres (60\%), sin embargo, cuando indagamos la experiencia de haber cocinado con el papá, resultó que los docentes hombres, tienen mayor porcentaje que las mujeres, reflejando $75 \%$ y $25 \%$ respectivamente. Destacamos que no se tienen datos perdidos, lo cual fortalece los análisis dado que todos los docentes respondieron estos ítems planteados.

Ante estos datos se confirma que se trata de un grupo de alumnos con elevado nivel de identificación con la cocina, por lo que era interesante conocer sus valoraciones en cuanto a las estrategias metodológicas que deberían tener sus docentes para el desarrollo de una formación adecuada en la especialidad de Gastronomía y lo hicimos, 
planteando afirmaciones en el instrumento específicamente en la dimensión-C. Algunos de los resultados expresados ante estas afirmaciones se muestran en la Tabla 5, haciendo la diferencia de la muestra de estudiantes en dos grupos: Grupo -A, alumnos que sus docentes si tienen la formación en nivel Superior de Educación y Grupo-B, alumnos que sus docentes no tienen esta formación Superior en Educación.

Tabla 5

Frecuencia de valoración emitida por la muestra de estudiantes en afirmaciones de la dimensión $C$ - estrategias metodológicas para el desarrollo de una formación adecuada en la especialidad de Gastronomía

\begin{tabular}{lcc}
\hline \multicolumn{1}{c}{ Ítems } & $\begin{array}{c}\text { Valoración en escala } \\
\text { de acuerdo }\end{array}$ \\
\cline { 2 - 3 } & Grupo A & Grupo B \\
\hline $\begin{array}{l}\text { C.2. El profesor relaciona los conocimientos teóricos de la asignatura con experiencias previas de } \\
\text { nuestras vidas. }\end{array}$ & $92.00 \%$ & $94.00 \%$ \\
$\begin{array}{l}\text { C.3. Los talleres prácticos que me dan son necesarios para mi formación profesional de la } \\
\text { Gastronomía. }\end{array}$ & $93.06 \%$ & $93.85 \%$ \\
$\begin{array}{l}\text { C.11. Esta asignatura se aprende sólo con la teoría. } \\
\text { C.16. La Gastronomía nacional nos la deberían enseñarse desde que somos niños. }\end{array}$ & $8.33 \%$ & $17.69 \%$ \\
$\begin{array}{l}\text { C.18. La mejor forma de aprender sobre Turismo y Gastronomía es el compromiso individual por } \\
\text { aprender. }\end{array}$ & $55.56 \%$ & $30.77 \%$ \\
\hline
\end{tabular}

En cuanto al dominio de los conocimientos teóricos por parte del docente (Tabla 5) vemos que los alumnos lo valoran con elevado porcentaje de aceptación (C.2), por lo que buscamos conocer también la valoración que dan los docentes a la afirmación planteada diciendo que las clases magistrales o expositivas de sus asignaturas son indispensables para los alumnos de la escuela de Gastronomía. Se ha encontrado que de los docentes que, si tienen la formación Superior en Educación, el 100\% están totalmente de acuerdo; en cuanto que los docentes que no tiene esta formación Superior en Educación, únicamente el $91.6 \%$ lo está e incluso el 8.4\% no está de acuerdo con la necesidad del dominio disciplinar de sus asignaturas.

Siendo que las estrategias metodológicas son elementos pedagógicos claves para el desarrollo de una formación adecuada en la especialidad de gastronomía, hemos buscado explicar la configuración de las correlaciones dentro de un conjunto de variables observadas en la dimensión de Estrategias metodológicas para el desarrollo de una formación adecuada en la especialidad de gastronomía y para las muestras de estudiantes, hemos usado el análisis factorial para inspeccionar e intentar identificar variables subyacentes o factores, reduciendo los datos a un pequeño número de factores que expliquen la mayor parte de la varianza observada en esta dimensión.

En este sentido, hemos aplicado la Medida Prueba de KMO (Kaiser-MeyerOlkin) de adecuación de muestreo y la prueba de esfericidad de Bartlett, destacando lo siguiente:

- Para el grupo de alumnos que sus docentes sí tienen la formación Superior en Educación, se identificaron 3 componentes principales de extracción, lo cual indica que 9 de los 20 ítems de esta dimensión son los que mejor explican los hallazgos y con el uso del Método de rotación: Promax con normalización Kaiser, 
obtenemos la distribución de ítems por componente, según se muestra en la Figura 6.

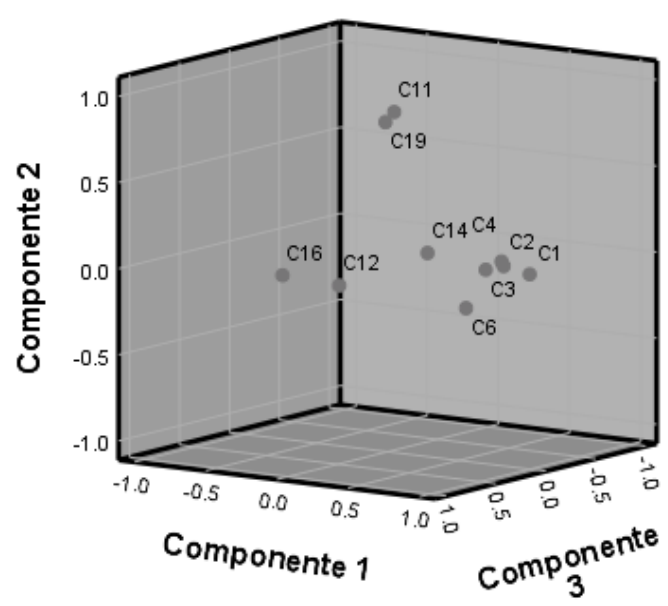

Figura 6. Distribución de ítem por componente dimensión C, de la muestra estudiantil de docentes con formación en Educación

- Para el grupo de alumnos que sus docentes no tienen formación Superior en Educación y bajo la aplicación de las mismas pruebas se identificaron 6 componentes principales de extracción, lo cual indica que 15 de los 20 ítems de esta dimensión son los que mejor explican los hallazgos, con base en el Método de rotación: Promax con normalización Kaiser.

- $\quad$ Para el grupo de docentes, una de las variables tiene una varianza cero y sólo hay una variable en el análisis, por lo que la dimensión "D" que corresponde a las estrategias metodológicas no tiene factores que expliquen y en este caso solo usamos un conjunto de respuestas múltiples para hacer la clasificación de todas las respuestas que explican toda la dimensión.

En vista de los análisis realizados a los resultados obtenidos, se resume que:

1. Las pruebas descriptivas aplicadas confirman a la Escuela de Gastronomía que sus alumnos ya tienen experiencias previas de cocina y que, si bien éstas han sido de forma empírica en sus casas, representan un reto pedagógico para los docentes porque están frente a un estudiante que ya tiene ciertos aprendizajes.

2. Para los dos subgrupos de la muestra de alumnos, los ítems priorizados que mejor explican la dimensión de las Estrategias metodológicas, son:

C.2.El profesor relaciona los conocimientos teóricos de la asignatura con experiencias previas de nuestras vidas.

C3. Los talleres prácticos que me dan son necesarios para mi formación como profesional de la Gastronomía.

C.11.Esta asignatura se aprende sólo con la teoría.

C.16. La Gastronomía nacional nos la deberían enseñarse desde que somos niños, $\mathrm{y}$ 
C.19. El Turismo Gastronómico se puede simular dentro de un contexto de 4 paredes del salón de clases.

3. Las pruebas descriptivas confirman que, los alumnos se muestran totalmente de acuerdo con esos ítems priorizados, excepto en el ítem C.11 referido al aprendizaje solo con teoría, en el cual el grupo de alumnos que sus docentes sí tienen formación Superior en Educación, únicamente el $8 \%$ está totalmente de acuerdo comparado con el $18 \%$ del otro grupo de alumnos.

4. Se pone en evidencia la coherencia de la técnica pedagógica de aprendizaje en la práctica, porque el ítem referido a los talleres prácticos que se dan a los alumnos son necesarios para la formación como profesional de la Gastronomía presenta una valoración del $80 \%$ en la prueba de KMO y esfericidad de Bartlett por parte de los estudiantes y se complementa con las respuestas de los docentes ya que el $100 \%$ de los docentes están totalmente de acuerdo.

5. La prueba de KMO y esfericidad de Bartlett aplicada en el ítem C.16 referido a que la Gastronomía nacional debería enseñarse desde que son niños, confirma altas valoraciones de aceptación de esta afirmación por parte de los dos subgrupos de la muestra de alumnos (docentes con o sin formación Superior en Educación) obteniéndose un $90 \%$ y un $70 \%$ respectivamente.

6. Respecto al ítem referido a que el Turismo Gastronómico se puede simular dentro de un contexto de 4 paredes del salón de clases, los dos subgrupos de la muestra de alumnos (con docentes con formación superior y sin ella) presenta altas valoraciones en la prueba de KMO y esfericidad de Bartlett ( $80 \%$ y $70 \%$ respectivamente), por lo que este ítem C-19 explica muy bien la dimensión estrategias metodológicas para el desarrollo de una formación adecuada en la especialidad de Gastronomía.

7. En la muestra de docentes, el mismo ítem referido a simulación del Turismo Gastronómico, es interesante apuntar que los docentes que no tienen formación Superior se muestran bastante de acuerdo en un $75 \%$; sin embargo, cuando la prueba mezcla los resultados de los docentes que sí tienen formación Superior en Educación, el porcentaje de bastante acuerdo disminuye a un $57.1 \%$.

\section{Discusión y conclusiones}

En cuanto a las condiciones que rodean el desarrollo de aprendizajes relacionados al Turismo Cultural Gastronómico en la Escuela de Gastronomía, podemos afirmar que las experiencias previas que han tenido los alumnos (independiente del género) habiendo cocinado anteriormente con sus abuelos y/o padres, se ve reflejado estadísticamente, porque afirman que la Gastronomía nacional se debería enseñar desde que son niños y destacamos que los alumnos de docentes que sí tienen formación Superior en Educación son los que se expresan totalmente de acuerdo para este planteamiento. En esta línea de formación del profesorado, es que Antoli et al. (2016) señaló que la formación del docente debía incluir aspectos de formación en valores culturales y en este caso, valores vinculados al arraigo culinario transferido de forma oral dentro de la familia.

$\mathrm{Al}$ contrastar estos resultados de los alumnos con los resultados expresados por los docentes, podemos decir que los docentes con formación Superior en Educación, también lo han podido identificar porque expresan desacuerdo ante la afirmación que la mejor forma de aprender sobre Turismo y Gastronomía es el compromiso individual que tiene cada estudiante para aprender, y valoran la experiencia previa que han tenido, 
en contraste con los docentes que no tienen esta formación Superior en Educación, ya que no valoran la experiencia previa que trae el alumno a la Escuela y únicamente valoran el momento del alumno dentro de su asignatura en la Escuela. Es aquí, donde se sustenta la necesidad de formación pedagógica del docente para poder trabajar partiendo del conocimiento previo que traen los estudiantes, así como fue señalado por Yánez et al. (2017) quien considera que, la creación de riqueza se hace desde el talento y conocimiento humano.

Cuando buscamos dar respuesta a la pregunta de investigación referida a saber si ¿es necesaria una didáctica universitaria específica en la promoción de competencias relacionadas con el Turismo Cultural Gastronómico en el campo de la formación Técnica Superior en Gastronomía?, podemos decir que todos los docentes muestran acuerdo en que los talleres prácticos son necesarios para la formación de los alumnos y se destaca lo siguiente:

1. El trabajo que realizan los docentes con formación Superior en Educación ha logrado permear en sus alumnos la necesidad de los talleres prácticos, porque sus estudiantes no están de acuerdo en que la asignatura se aprende solo con la teoría, en contraste con los alumnos que sus docentes no tienen esta formación Superior en Educación ya que valoran que la teoría es suficiente para aprender, y

2. Solamente los docentes con formación Superior en Educación no están totalmente de acuerdo en que el Turismo Gastronómico se puede simular dentro de un contexto de 4 paredes del salón de clases, en virtud de lo que implica el aprendizaje por medio de la simulación o creación de realidades aumentadas y la formación pedagógica que se requiere para poder hacerlo. Esto va en sintonía con los aportes que dieron Franco y Pmienta et al. (2016) porque el foco de la Didáctica es movilizar al estudiante para elaborar, construir y reconstruir conocimientos, por eejemplo,en la simulación.

En definitiva, los resultados estadísticos presentados únicamente para la dimensión Estrategias metodológicas para el desarrollo de una formación adecuada en la especialidad de gastronomía, dejan en evidencia los efectos del acto didáctico docente sobre el tipo de aprendizajes que promueven en alumnos de Educación Superior del sector Turismo Cultural Gastronómico que son: a) la aplicación de la teoría a la experiencia previa del alumno genera mayor aprendizaje técnico así como, b) la puesta en práctica de la teoría en talleres y la simulación de realidades aumentadas en salón de clases genera conductas requeridas pero se necesita formación pedagógica para poderlo simular. Por lo tanto, es necesario ahondar en investigaciones referidas a la calidad de la formación del docente, para prepararlo a trabajar en carreras Educación Superior de Turismo y afines. No es suficiente sólo dominar elementos cognitivos de la asignatura, sino que, el docente debe aplicar además, los ingredientes culturales propios y de sus alumnos, para hacer la traducción pedagógica hacia las conductas requeridas en el sector del Turismo Cultural Gastronómico.

\section{Referencias}

Antoli, V. B. (2016). Desarrollo profesional del profesor universitario. Campo Abierto. Revista de Educación, 10(1), 175-197. Retrieved from file:///C:/Users/portatil/Downloads/2622-Texto\%20de1\%20art\%C3\%ADculo9150-1-10-20160426.pdf 
Ballesteros, J. (2016). El aprendizaje basado en proyectos y su incidencia en la formación práctica en la disciplina de autotrónica de los estudiantes del Instituto Tecnológico Superior Guayaquil. Retrieved from: http://repositorio.uta.edu.ec/jspui/handle/123456789/22484

Benito, M. (2014). Estudio exploratorio sobre la interpretación didáctica del arte en el museo a través de tecnologías móviles (Tesis Doctoral). Universitat de Barclona, Barcelona. Retrieved from: http://diposit.ub.edu/dspace/bitstream/2445/54240/1/01.MVLB_TESIS.pdf

Bosch, M (2000). Un punto de vista antropológico: la evolución de los "elementos de representación" en la actividad matemática. En N.A. Climent Rodríguez, L.C. Contreras, y J. Carrillo (Eds.), Cuarto Simposio de la Sociedad Española de Investigación en Educación Matemática (15-28). Huelva: Sociedad Española de Investigación en Educación Matemática, SEIEM. Retrieved from: http://funes.uniandes.edu.co/1427/1/Bosch2003Un_SEIEM_15.pdf

De Oliveira R., R., y De Moura A., M. C. (2016). A relevância da formação didáticopedagógica na docência universitária. Revista Evidência, 12(12), 45-58. Retrieved from: file:///C:/Users/portatil/Downloads/496-1904-1-PB.pdf

Devesa, M. (2013). Didáctica de la armonía: Una propuesta de enseñanza basada en el aprendizaje significativo. (Tesis Doctoral). Universitat d'Alacant-Universidad de Alicante, Alicante. Retrieved from: http://hdl.handle.net/10045/41199

Franco, M., y Pimenta, S. (2016). Didática multidimensional: por uma sistematização conceitual. Educação y Sociedade, 37(135), 539-553. doi: 10.1590/ES010173302016136048

Gómez, M. (2009). Introducción a la metodología de la investigación científica. Argentina: BRUJAS. Retrieved from: http://blogs.unlp.edu.ar/seminariofm2/files/2017/04/Gomez-Cap3-4.pdf

Iszoro, E. (2015). Métodos directos de patronaje creativo: didáctica y experimentación. (Tesis Doctoral). Universidad Politécnica de Madrid. Escuela Técnica Superior de Arquitectura, Madrid. Retrieved from: http://oa.upm.es/42727/1/EVA_ISZORO_ZAK.pdf

Marhuenda, F. y Ros-Garrido, A. (2015). What sense can we make of the possibility of vocational didactics? An approach from the Spanish school-based system complemented by non-formal vocational training. International journal for research in Vocational Education and Training, 2(3), 170-181. Retrieved from: urn:nbn:de:0111-pedocs-115574

Mirzagitova, A. L., y Akhmetov, L. G. (2016). Formation of the Professional and Didactic Culture of the Future Teacher. International Journal of Environmental and Science Education, 11(14), 58-74. Retrieved from: https://www.um.edu.mt/library/oar//handle/123456789/31339

Pacheco, L. (2013). La Didáctica Universitaria en el Contexto de la Andragogía: Aprender a Aprender en la Educación de Personas Adultas. TEC Empresarial, 2(2), 29-33. Retrieved from: http://revistas.tec.ac.cr/index.php/tec_empresarial/article/view/768

Pavo, H., y Ángel, M. (2014). La construcción conjunta de conocimiento práctico en la formación inicial de los maestros: un análisis de la interacción en tono a los dilemas docentes en contextos colaborativos virtuales. (Tesis Doctoral). Universitat Oberta de Catalunya. Internet Interdisciplinary Institute (IN3). Retrieved from: http://hdl.handle.net/10803/305492 
Porro, S. (2015). Especialización en Pedagogía y Didáctica Universitaria. (Trabajo Final Integrador). Universidad Nacional de Quilmes, Argentina. Retrieved from: http://ridaa.demo.unq.edu.ar

Sauthier, K. D., y Dos Santos J. K. (2016). Pressupostos teóricos e percepções docentes em torno da Didática. Ensino \& Pesquisa-Revista Multidisciplinar de Licenciatura e Formação Docente, 14(1), 13-24. Retrieved from: file://C:/Users/portatil/Downloads/405-2516-1-PB.pdf

Torres, M. M. (2017). El controvertido desarrollo de la didáctica general, las didácticas específicas y su aporte a la didáctica universitaria. Pedagogía y saberes, (28), 115-121. doi: 10.17227/01212494.28pys115.121

Yánez, D. X. (2017). Programa de Formación Docente para la Enseñanza en la Educación Superior. (Tesis Doctoral). Universidad de Guayaquil. Dirección de Posgrado, Guayalqui. Retrieved from: http://repositorio.ug.edu.ec/handle/redug/21998

Zhou, X. (2015). La pasiva en español y su enseñanza a estudiantes chinos: análisis contrastivo, análisis de errores y propuestas didácticas. (Tesis Doctoral) Universidad Complutense de Madrid, Madrid. Retrieved from: https://eprints.ucm.es/33239/1/T36406.pdf

Fecha de recepción: 28/12/2018

Fecha de revisión: 30/05/2019

Fecha de aceptación: 15/07/2019 